\title{
43
}

\section{Optimized dual-view light sheet microscopy for large area cleared specimens}

Vladislav Gavryusev $^{1,2}$, Giacomo Mazzamuto ${ }^{3,2}$, Giuseppe Sancataldo $^{4}$, Luca Pesce ${ }^{1,2}$, Annunziatina Laurino ${ }^{5}$, Marina Scardigli $^{1,2}$, Irene Costantini ${ }^{3,2}$, Ludovico Silvestri ${ }^{1,2,3}$, Francesco Saverio Pavone ${ }^{1,2,3}$

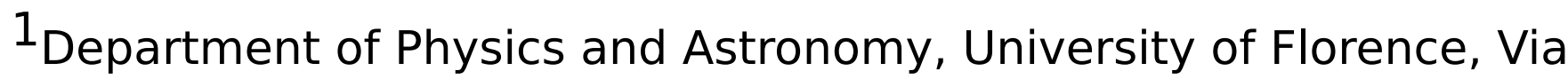
G. Sansone, 1, 50019 Sesto Fiorentino (FI), Italy. ${ }^{2}$ European Laboratory for Non-linear Spectroscopy, Via Nello Carrara, 1, 50019 Sesto Fiorentino (FI), Italy. ${ }^{3}$ National Institute of Optics, National Research Council, Via Nello Carrara, 1, 50019 Sesto Fiorentino (FI), Italy. ${ }^{4}$ Department of Physics and Chemistry, University of Palermo, Viale delle Scienze, 17, 90128, Palermo, Italy. ${ }^{5}$ Department of Neuroscience, Psychology, Drug Research and Child Health, University of Florence, Viale Pieraccini, 6, 50139 Florence, Italy

\section{Abstract Text}

A key challenge in modern neuroscience is represented by the volumetric mapping of the human brain neuronal architecture. Towards this goal, Light Sheet Fluorescence Microscopy (LSFM) enables to investigate its structure from the sub-cellular to the organ scale, by recording the neuronal population distributions in large cleared tissue samples with micrometric resolution and within accessible experimental time frames. In order to image specimens up to tens of $\mathrm{cm}$ in lateral size with minimal physical sectioning and to avoid introducing significant optical aberrations due to refractive index mismatch between the tissue and the surrounding medium, both the sample and detection objective have to be placed in a large reservoir filled with a refractive index matching medium. Fulfilling such requirements demands a custom-made and optimized LSFM for imaging and a laborious sample preparation. 
Here, we present a custom-built dual-view inverted confocal light sheet fluorescence microscope (di2CLSFM) [1]. This instrument captures two orthogonal views of the sample at the same time in order to obtain an almost isotropic micrometric resolution along the three optical axes through computational fusion, concurrently providing a state-of-the-art volumetric acquisition speed of 0.5 $\mathrm{cm}^{3} /$ hour. Up to four fluorescence bands can be recorded sequentially on the two sCMOS cameras, selected through appropriate band-pass filters housed on a motorized filter wheel and matched with the appropriate laser illumination wavelength by an acousto-optical tunable filter. The inverted microscope geometry allows to accommodate samples with diameter up to $30 \mathrm{~cm}$ or to place multiple centimeter sized specimens in a custom-built sealed fused silica holder for consecutive imaging and long-term storage. We also present our results on volumetric fluorescence imaging of several different, large and optically cleared samples and our image handling and analysis pipeline, designed to process multi-Terabyte-sized datasets and to obtain cell type-specific neuronal spatial distributions through a robust and automatic artificial intelligence-assisted segmentation [2]. 